\title{
Longitudinal Evidence for Functional Specialization of the Neural Circuit Supporting Working Memory in the Human Brain
}

\author{
Amy S. Finn, ${ }^{1}$ Margaret A. Sheridan, ${ }^{2,3}$ Carla L. Hudson Kam, ${ }^{1}$ Stephen Hinshaw, ${ }^{1}$ and Mark D'Esposito ${ }^{1,4}$ \\ ${ }^{1}$ Department of Psychology, University of California, Berkeley, Berkeley, California 94720-1650, ${ }^{2}$ Harvard University School of Public Health, Boston, \\ Massachusetts 02215, 32Developmental Medicine Center Laboratory of Cognitive Neuroscience, Children's Hospital Boston, Boston, Massachusetts 02215- \\ 5365, and ${ }^{4}$ Helen Wills Neuroscience Institute, University of California, Berkeley, Berkeley, California 94720-3190
}

\begin{abstract}
Although children perform more poorly than adults on many cognitive measures, they are better able to learn things such as language and music. These differences could result from the delayed specialization of neural circuits and asynchronies in the maturation of neural substrates required for learning. Working memory - the ability to hold information in mind that is no longer present in the environment-comprises a set of cognitive processes required for many, if not all, forms of learning. A critical neural substrate for working memory (the prefrontal cortex) continues to mature through early adulthood. What are the functional consequences of this late maturation for working memory? Using a longitudinal design, we show that although individuals recruit prefrontal cortex as expected during both early and late adolescence during a working memory task, this recruitment is correlated with behavior only in late adolescence. The hippocampus is also recruited, but only during early, and not late, adolescence. Moreover, the hippocampus and prefrontal cortex are coactive in early adolescence regardless of task demands or performance, in contrast to the pattern seen in late adolescents and adults, when these regions are coactive only under high task demands. Together, these data demonstrate that neural circuitry underlying working memory changes during adolescent development. The diminishing contribution of the hippocampus in working memory function with age is an important observation that informs questions about how children and adults learn differently.
\end{abstract}

\section{Introduction}

Working memory (WM) comprises a set of cognitive processes required for many, if not all, forms of learning (Bayliss et al., 2005; Cowan, 2005). This ability has been shown to be important for myriad cognitive abilities (Conway et al., 2003; Unsworth et al., 2009) and scholastic achievement (Bayliss et al., 2005). The neural substrates supporting WM undergo changes well into adulthood (Sowell et al., 2004; Gogtay et al., 2006; Lenroot and Giedd, 2006), within both regions that are necessary for WM in adults-the prefrontal cortex (PFC) (Goldman-Rakic, 1987; Curtis and D'Esposito, 2003)—and regions that are recruited only during specific contexts-the hippocampus (Ranganath and Blumenfeld, 2005). Using longitudinal functional magnetic resonance imaging (fMRI) in adolescents, we ask whether these anatomical changes observed in normal development are accompanied by changes in the functional WM circuit.

Adolescence is an important period of time to explore these changes for several reasons. First, adolescence is a critical period

Received Dec. 18, 2009; revised April 22, 2010; accepted June 27, 2010.

This research was funded by National Institutes of Health Grants MH63901 and NS40813 (M.D.) and a National Science Foundation Graduate Research Fellowships Program Award (A.S.F.). We thank Jesse Rissman and Dan Handwerker for advice regarding data analysis and Ashley Smart and Jessica Morrison for assistance with data processing.

Correspondence should be addressed to Amy S. Finn, Department of Psychology, University of California, Berkeley, 3210 Tolman Hall \#1650, Berkeley, CA 94720-1650. E-mail: amyfinn@berkeley.edu.

D0I:10.1523/JNEUROSCI.6266-09.2010

Copyright $\odot 2010$ the authors $\quad 0270-6474 / 10 / 3011062-06 \$ 15.00 / 0$ for PFC development (Lenroot and Giedd, 2006). Second, neurodevelopmental investigations generally report greater recruitment of brain regions involved in WM in adults than in children (Casey et al., 1995; Thomas et al., 1999; Klingberg et al., 2002; Tsujimoto et al., 2004; Crone et al., 2006; Scherf et al., 2006; Olesen et al., 2007; O'Hare et al., 2008; Thomason et al., 2009). Comparisons between adolescents and adults, however, are more varied, with some studies reporting few differences (Crone et al., 2006; O'Hare et al., 2008) and others reporting more diffuse brain activity during adolescence (Scherf et al., 2006; Olesen et al., 2007). We aim to resolve these discrepancies by examining changes in the same adolescents across two time periods. Third, there is growing evidence that adolescence is a time of substantial reorganization on a network level (Fair et al., 2007). Thus, multivariate analyses of fMRI can illuminate whether the functional circuit supporting WM function also undergoes reorganization, something not addressed in previous developmental investigations. Finally, few behavioral differences are observed between adolescents and adults on simple WM tasks (Luciana and Nelson, 1998; Gathercole, 1999; Cowan et al., 2003; Luna et al., 2004; Davidson et al., 2006) despite the PFC's relative immaturity at this age, suggesting the involvement of additional regions during the engagement of WM processes. Illuminating any such differences could help explain why a behavioral difference is not observed. The hippocampus is a likely candidate. In adults, the hippocampus is recruited during WM tasks only when stimuli are novel or relations between stimuli must be formed (Mitchell et 


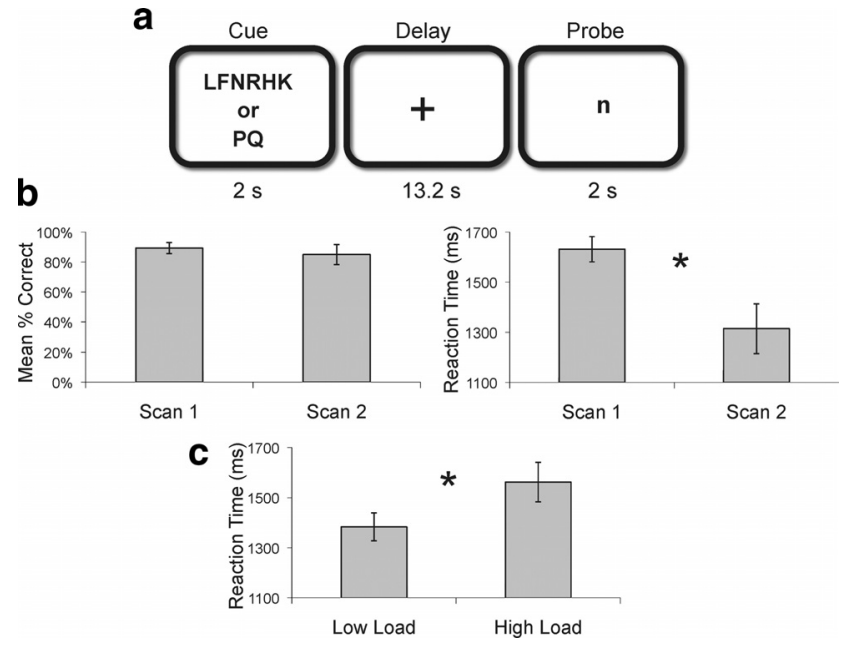

Figure 1. Experimental task and behavioral data. $\boldsymbol{a}$, Participants encoded either 2 or 6 letters (cue) and retained them across a $13.2 \mathrm{~s}$ delay interval. When a probe was presented, they then determined whether or not a single letter was part of the memory set ( $2 \mathrm{~s}) . \boldsymbol{b}, \boldsymbol{c}$, Accuracy and RTs across scan times $(\boldsymbol{b})$ and memory loads (c). Error bars represent SEM.

al., 2000; Ranganath and D'Esposito, 2001; Piekema et al., 2006). Likewise, patients and animals with hippocampal damage are impaired on WM tasks involving complex and novel objects (e.g., Buffalo et al., 1998; Eacott et al., 1994; Hannula et al., 2006).

The present investigation therefore addresses the following specific questions: (1) Does the WM network change during adolescence? (2) Moreover, is the hippocampus involved in the WM network during early, but not later, adolescence?

\section{Materials and Methods}

Participants and procedure. We used fMRI and followed 10 female adolescents longitudinally [mean age 15.1 years (SD: 1.55) for scan 1 (S1) and 18.3 years (SD: 1.45) for scan 2 (S2); all were right-handed, native English speakers]. We included only females since gender differences in developmental trajectories have been reported, especially during adolescence and for subcortical structures (Lenroot and Giedd, 2010). Participants were excluded if they reported any of the following: a history of brain trauma, birth mother drug or alcohol use during pregnancy, taking psychoactive medication, frequent use of drugs or alcohol, having metal on or near the body, or any other health condition relevant for scanning.

During both scans, we administered a delayed match-to-sample task (Sternberg, 1969; Rypma and D'Esposito, 2000) in which participants encoded either 2 (low load) or 6 (high load) visually presented uppercase letters for $2 \mathrm{~s}$ (cue), held the letters in mind for a $13.2 \mathrm{~s}$ (delay) period and then responded as to whether a lowercase letter presented at test (probe) matched one of the encoded letters (Fig. 1a). There were a total of 80 trials (50\% high load; $50 \%$ low load, $50 \%$ where the probe letter matched a letter in the encoding set; randomized across trials).

fMRI data acquisition and analysis. Functional MRI data were acquired using a 4.0 T Varian INOVA MR scanner. Functional data were obtained using a two-shot echo-planar imaging (EPI) sequence sensitive to BOLD contrast (effective $\mathrm{TR}=2200 \mathrm{~ms}, \mathrm{TE}=28 \mathrm{~ms}, \mathrm{FOV}=$ $22.4 \mathrm{~cm}^{2}$, matrix size $\left.=64 \times 64\right)$. Image processing and univariate and multivariate analysis were completed using SPM2 (Friston et al., 1991). Functional MRI data processing included a linear-time interpolation algorithm to double the effective sampling rate. Temporal sync interpolation was used to correct for between-slice timing differences in image acquisition. Data were spatial smoothed using 10 mm FWHM Gaussian kernel. Motion correction was accomplished using a six-parameter rigid-body transformation algorithm (Friston et al., 1995a). A high-resolution 3D T1-weighted structural scan (MPFLASH sequence) and an in-plane high-resolution T2-weighted structural scan (GEMS sequence) were acquired for anatomical local- ization and normalization purposes. A total of 1230 whole-brain volumes were collected for each subject. The first 10 scans were dropped from the beginning of every run to account for gradient instabilities, resulting in 1130 usable whole-brain volumes per participant. For each participant, total time in the scanner was $\sim 1 \mathrm{~h}, 30 \mathrm{~min}$ for each scan.

For every participant, any run that included $5.5 \mathrm{~mm}$ or more movement in any of the six parameters used for movement correction $(x, y, z$, roll, pitch, and yaw) was excluded from the analysis. This exclusion criterion allowed us to retain a large number of trials while restricting movement within an activated slice. Mean movement parameters after the exclusion criteria were applied were very small and did not differ across scan sessions $\left[\mathrm{S} 1\right.$ mean $(\mathrm{M})=0.117 \mathrm{~mm}(\mathrm{SD}=0.09), \mathrm{M}=0.003^{\circ}$ $(\mathrm{SD}=0.005) ; \mathrm{S} 2 \mathrm{M}=0.133 \mathrm{~mm}(\mathrm{SD}=0.162), \mathrm{M}=0.002^{\circ}(\mathrm{SD}=$ $0.002)$ ]. To additionally account for subject movement, movement correction parameters were included as covariates in each individual's general linear model analysis. As a result, several adolescents had $<1130$ acquisitions. The smallest number of acquisitions allowed was 452 , for one participant. This participant performed 40 task trials out of a possible 80 , and this was during their first scanning session.

Before statistical analyses, data were normalized to Montreal Neurological Institute (MNI) space, As described previously (Sheridan et al., 2007), a statistical parametric map was calculated for each participant based on linear combinations of the regressors modeling each task period (Friston et al., 1995b). Regressors were convolved with the canonical hemodynamic response function provided by SPM 2 and defined for each stage (cue, delay, and probe) of each task condition. Only trials with correct responses were incorporated in the analysis. Individual results were then combined into a group analysis; paired $t$ tests were used for across-scan comparisons.

To compute functional connectivity, a unique parameter estimate ( $\beta$ value) for the events in each trial was computed for each participant and then sorted by task period (i.e., cue, delay, and probe), yielding a $\beta$ series. The extent to which two regions interact is quantified by the extent to which their respective $\beta$ series are correlated (Rissman et al., 2004). Regions of interest (ROIs) for both brain-behavior and connectivity analyses were constructed from these group data by choosing the 10 most active, contiguous voxels within the PFC (all participants at both scan times, $p<0.001$ ) or hippocampus (all participants at $S 1, p<0.001$ ). Our definition of the lateral PFC included the middle and inferior frontal gyri, ventral to the superior frontal sulcus and anterior to the precentral sulcus. Contrast values were extracted using MarsBar (Brett et al., 2002).

All correlation analyses were conducted using Matlab 6.5 (http://www. mathworks.com). To allow statistical conclusions to be made based on the correlation magnitudes, we applied an arc-hyperbolic tangent transform (Fisher, 1921) to the correlation coefficients of all brain voxels. Since the correlation coefficient is inherently restricted to range from -1 to +1 , this transformation serves to make its null hypothesis sampling distribution approach that of the normal distribution. The transformed correlation coefficients were then divided by their known SD to yield $z$ scores. Group level random-effects $t$ tests were then conducted to identify voxels for which the mean of subjects' transformed correlation coefficients was reliably greater than zero.

\section{Results}

Accuracy did not differ across the two scans $\left(t_{(9)}=0.562, p=\right.$ 0.588). Consistent with many developmental studies using reaction time (RT) (e.g., Sheppard and Vernon, 2008), participants were faster to respond when they were older (i.e., at S2, $t_{(9)}=$ 3.658, $p=0.005)$. During both scans, participants were more accurate $\left(t_{(9)}=2.63, p=0.027\right)$ and faster to respond $\left(t_{(9)}=\right.$ $-4.678, p=0.001)$ at low load (2 vs 6 letters).

Neural recruitment was assessed for all task periods (e.g., cue, delay, and probe). Consistent with many other investigations of WM, lateral PFC activity was observed during all stages of the task at both scan times (S1 and S2) (supplemental Tables 1, 2, available at www.jneurosci.org as supplemental material). Additionally and in accordance with our hypothesis, participants recruited 
hippocampus during $\mathrm{S} 1$, but not $\mathrm{S} 2$, for all three task periods (cue, delay, and probe) (supplemental Tables 1, 2, available at www.jneurosci.org as supplemental material). Accordingly, hippocampal activity was greater during S1 than S2 during all three task periods for each load condition and collapsed across loads (see Fig. 2 and supplemental Table 3, available at www. jneurosci.org as supplemental material).

These results suggest that while adolescents recruit PFC as expected during a WM task, younger adolescents additionally recruit the hippocampus. This is the first demonstration of hippocampal activity for any age group during a WM task that does not include complex/novel or location-bound objects. Given that neither adults (Rypma and D'Esposito, 2000) nor these same individuals 3 years later recruit the hippocampus for this task, these data show that neural recruitment during WM function is qualitatively different earlier in adolescence. Why do individuals recruit the hippocampus when they are younger but not when they are older? This could be a consequence of several, not entirely separate, factors, such as differences in task difficulty during each of the scan times and differences in developmental trajectories of the hippocampus and PFC. These possibilities are explored in turn.

We explored task difficulty in two ways: first, by examining neural recruitment for high versus low memory loads, and second, by comparing recruitment across scan times on a subset of trials that were matched for RT. Regions that are recruited to a greater extent with increasing load should reflect increased task difficulty. As expected, there was greater recruitment of frontal regions during all task periods for high as compared to low loads during S1 (supplemental Table 4, available at www.jneurosci.org as supplemental material) and during the delay and probe periods for S2 (supplemental Table 5, available at www.jneurosci.org as supplemental material). However, load-related recruitment was not observed in the hippocampus at S1 or S2, suggesting that frontal, but not hippocampal, regions are sensitive to relative task difficulty.

Thus, it seems that the hippocampus is not recruited simply due the task being more difficult for younger adolescents. However, RTs were slower at S1 than S2. Thus, we compared the subset of trials in which RT was equated across the two time periods to determine whether the pattern of hippocampal activity we observed changed. In this analysis, we compared the fastest 75\% of trials during S1 (mean RT: 1398.5 ms; range: 2273-805 $\mathrm{ms}$ ) and the slowest 75\% of trials during S2 (mean RT: $1392.3 \mathrm{~ms}$; range: $3674-723 \mathrm{~ms}$ ). This analysis again revealed that hippocampal activity was greater during S1 than S2, as observed separately during the encoding and delay periods (supplemental Table 6, available at www.jneurosci.org as supplemental material). Thus, our primary result persists despite comparing trials at S1 with faster RTs, which could be considered easier, with trials at S2 with slower RTs.

The greater involvement of the hippocampus during performance of WM task at S1 could be due to the immaturity of the PFC that leads to compensatory recruitment of the hippocampus. Alternatively, it could simply reflect normal developmental

\section{Scan $1>\operatorname{Scan} 2$}

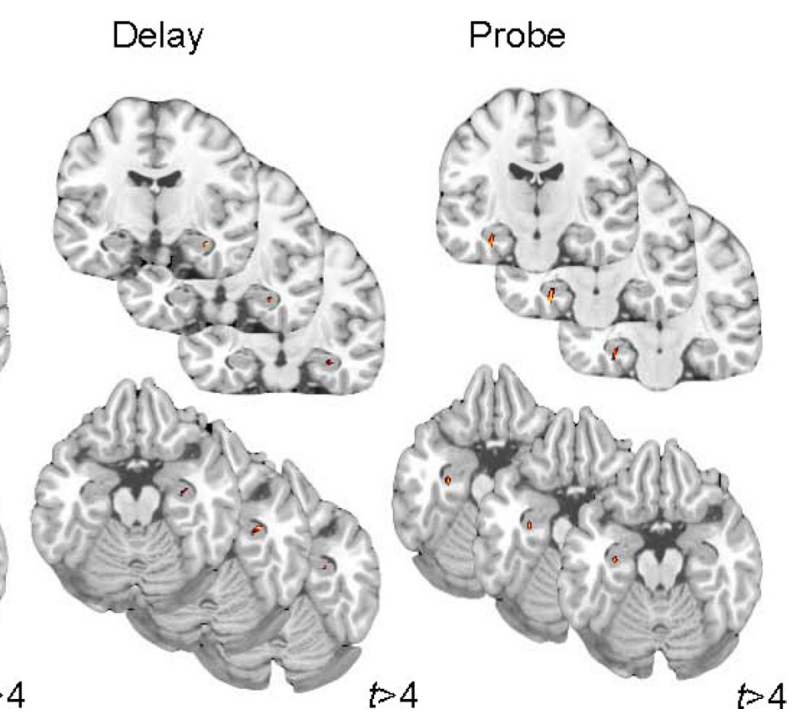

$>4$ differences in the trajectory of cortical maturation (hippocampal earlier, PFC later). To provide evidence that PFC's functional role in WM function is delayed in time, we explored the relationship between behavior and PFC function across scanning sessions. Across all participants, we therefore correlated left PFC activity during each task period with RT. We found that early in adolescence (S1), there was no significant association between PFC activity and RT for any of the task periods (encoding: $r=0.222$, $R^{2}=0.049, p=0.538$; delay: $r=-0.269, R^{2}=0.073, p=0.452$; probe: $r=-0.523, R^{2}=0.274, p=0.121$ collapsed across load). In contrast, at $\mathrm{S} 2$ the correlation was robust during encoding $(r=$ $-0.705, R^{2}=0.497, p=0.023$ ) (Fig. $3 a$ ), and in the predicted direction (though not significant) during other task periods. Moreover, there was a significant relationship between the change in the degree to which the PFC (S2-S1) was recruited and change in reaction time (S1-S2) across scan times for encoding and delay periods (cue: $r=-0.804, R^{2}=0.646, p=0.005$; delay: $r=-0.653, R^{2}=0.462, p=0.041$ ), showing that adolescents who recruited the PFC more during the second than during the first scan were the ones whose RTs improved the most across scan times (Fig. 3b). Thus, data show a tight link between PFC function and behavior at S2 but not S1, suggesting that the functional role of the PFC is developmentally delayed.

To investigate the role of the hippocampus in the WM network directly, we measured functional connectivity between the hippocampus and PFC at both scan times. We chose to focus this analysis on the encoding period because $S 1$ hippocampal recruitment is most robust during encoding; and this is also the task phase during which reliable brain-behavior associations were observed at S2. First, we examined functional connectivity (Rissman et al., 2004) between the right hippocampus and the rest of the brain at $S 1$. To directly compare connectivity at S1 and S2, we also measured functional connectivity of the left PFC region used above and the rest of the brain. At S1, activity in the right hippocampus was functionally correlated with bilateral regions in the lateral PFC (left: $t=4.40, p<0.0001$; right: $t=8.39, p<$ 0.0001 ) and the contralateral hippocampus (left: $t=4.12, p<$ 0.0001 ) (Fig. 4; supplemental Table 9, available at www.jneurosci. org as supplemental material). Also at S1, activity in the left PFC 


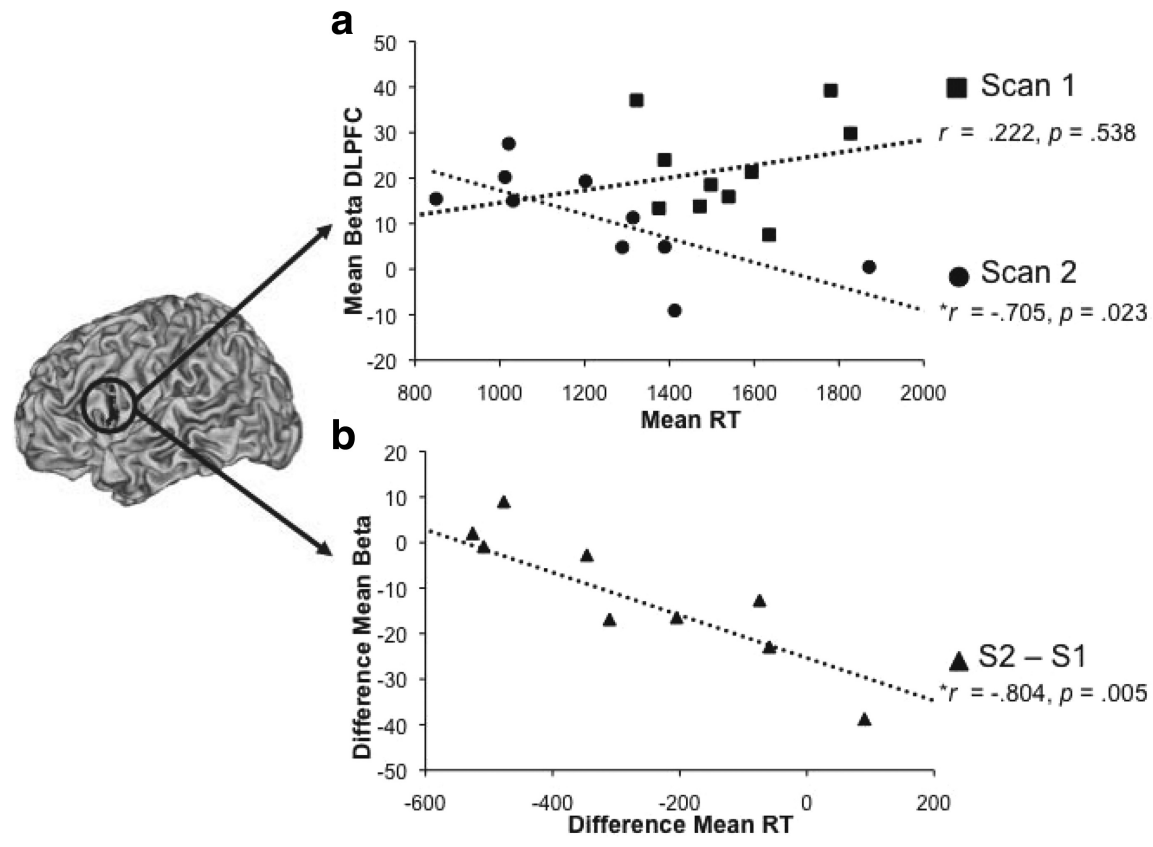

Figure 3. Brain-behavior correlations. $\boldsymbol{a}$, Mean $\beta$ values for each individual in left PFC during encoding are plotted against the mean RT for individuals separately for each scan time. $\boldsymbol{b}$, The difference across scan times in PFC $\beta$ values during encoding and RTs is plotted.

a Hippocampal seed
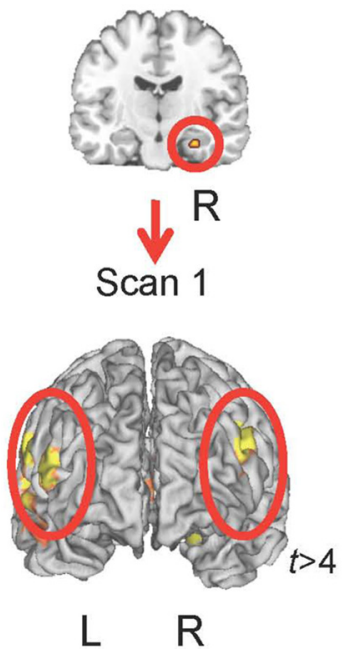

b
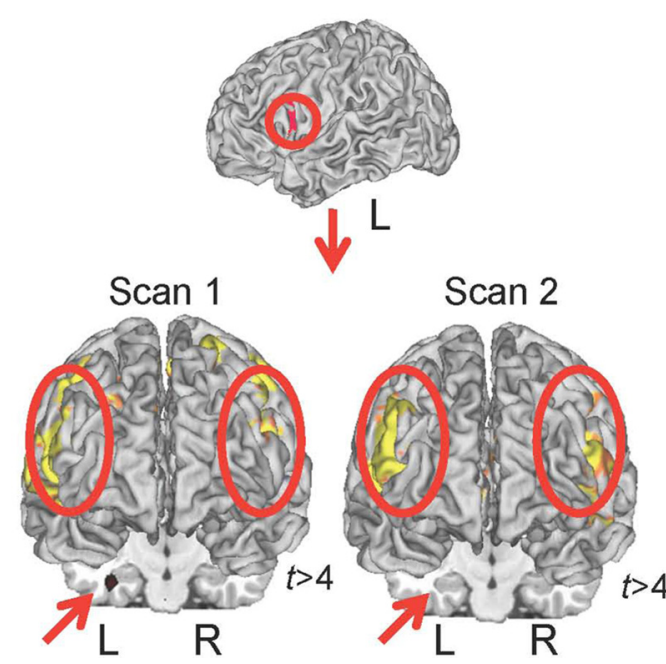

Figure 4. Hippocampal-PFC connectivity. $\boldsymbol{a}$, Hippocampal seed used in the connectivity analyses obtained during the encoding period in scan 1 . This seed was functionally correlated with bilateral lateral PFC, and contralateral hippocampus (not depicted) during scan 1. $\boldsymbol{b}$, Left PFC seed used in the connectivity analyses obtained during the encoding period across both scan times. This seed was functionally correlated with bilateral lateral PFC during both scan 1 and scan 2 but only the left hippocampus during scan 1.

was correlated with bilateral hippocampus (left: $t=4.67, p<$ 0.0001; right: $t=4.13, p<0.0001$ ) (Fig. 4; supplemental Table 7, available at www.jneurosci.org as supplemental material). Despite the strong association between hippocampal and PFC activity during S1, no such association was observed in the same individuals, performing the same task 3 years later. At S2, no significant correlations between PFC and hippocampus were observed (Fig. 4). Rather, the left PFC is correlated primarily with the contralateral right PFC (supplemental Table 8, available at www.jneurosci.org as supplemental material). Thus, the hip- pocampus appears to be part of the WM network during earlier stages of adolescence, but not later.

Previous work in adults, however, has shown that hippocampal connectivity with PFC and stimulus-selective posterior association cortex increased with high but not low loads (Rissman et al., 2008), or with difficult but not easy tasks. If individuals are more adult-like at S2, hippocampal-PFC connectivity might likewise be present for high but not low mnemonic loads. To test this hypothesis, we examined hippocampal-PFC connectivity for each scan time separately for high (6 letters) and low (2 letters) loads. As predicted given our reported findings, functional correlations between the left hippocampus and left PFC were significant regardless of load condition at S1 (low load: $t=4.53, p<0.0001$; high load: $t=5.02, p<0.0001)$. However, at S2, the left PFC and left hippocampus were correlated only during high load $(t=4.85$, $p<0.0001$ ).

To further explore the factor of task difficulty, we investigated whether RT would show a similar result. We split observations based on median reaction time (for both S1 and S2) and probed PFChippocampus connectivity for each load. The most difficult trials are those for which adolescents were slow to respond during a high mnemonic load. As in the previous analysis, functional correlations between the left hippocampus and left PFC were significant regardless of load or RT at S1 (fast/low: $t=2.77, p<0.005$; fast/high: $t=3.43, p<0.001$; slow/low: $t=3.17, p<0.001$; slow/high: $t=2.72$, $p<0.005)$. At S2, however, functional correlations were significant only during slow trials (low: $t=2.90, p<0.005$; high: $t=4.42, p<0.00005)$, an effect that was most salient during high load.

\section{Discussion}

In summary, this is the first longitudinal fMRI study demonstrating that functional network specificity changes across adolescence. Data show that the WM circuit becomes increasingly specialized with age. Earlier in adolescence, participants recruited both the lateral PFC and hippocampus during a WM task, but did not recruit the hippocampus 3 years later during performance of the same task. Moreover, whereas PFC and hippocampal activity are correlated regardless of WM load and behavior during early adolescence, these associations are observed only during high mnemonic loads 3 years later, a time during which the PFC is also predictive of behavior.

Our data aids in the understanding of the mismatch in the literature on the development of WM during adolescence. Behaviorally, basic WM maintenance processes show minimal changes 
during this time period (Luciana and Nelson, 1998; Gathercole, 1999; Cowan et al., 2003; Luna et al., 2004; Davidson et al., 2006), but a large body of evidence shows that the primary neural substrate for WM-the PFC - undergoes great structural and functional change during this same time (Sowell et al., 2004; Lenroot and Giedd, 2006; Thomason et al., 2009). By examining functional recruitment and network connectivity, we have shown that younger adolescents recruit an additional region, the hippocampus, during WM function. This recruitment could buttress WM function such that no behavioral differences are observed. Additional studies will be necessary to determine whether earlier hippocampal recruitment is compensatory or due to normal developmental differences in the trajectory of cortical maturation.

Interestingly, previous researchers have suggested that protracted PFC development might actually be a benefit, rather than a hindrance, to children; advanced PFC abilities are hypothesized to interfere with probability, convention, and imitative learning, forms of learning where children have been shown to outperform adults (Ramscar and Gitcho, 2007; Thompson-Schill et al., 2009). Here, we extend these previous theoretical formulations by demonstrating that the hippocampus is engaged in younger individuals during the performance of a WM task that does not engage the hippocampus in older individuals (see also Chatham et al., 2009). Because younger individuals appear to recruit the hippocampus in circumstances that adults do not, they might also bind, consolidate, store, and retrieve information in more situations than adults. Thus, we suggest that this hippocampal involvement in the broader WM network-and not just delayed PFC maturation-might actually be a crucial part of children's learning differences. Qualitative differences in the networks younger individuals employ to achieve basic aspects of cognition such as WM therefore hold the promise of helping us understand age-related learning differences we observe in nature.

\section{References}

Bayliss DM, Jarrold C, Baddeley AD, Gunn DM (2005) The relationship between short-term memory and working memory: complex span made simple? Memory 13:414-421.

Brett M, Anton J, Valabregue R, Poline J (2002) Region of interest analysis an SPM toolbox. In: Functional mapping of the human brain. Sendai, Japan: Available on CD-ROM in Neuroimage, Vol 16, No 2.

Buffalo EA, Reber PJ, Squire LR (1998) The human perirhinal cortex and recognition memory. Hippocampus 8:330-339.

Casey BJ, Cohen JD, Jezzard P, Turner R, Noll DC, Trainor RJ, Giedd J, Kaysen D, Hertz-Pannier L, Rapoport JL (1995) Activation of prefrontal cortex in children during a nonspatial working memory task with functional MRI. Neuroimage 2:221-229.

Chatham CH, Frank MJ, Munakata Y (2009) Pupillometric and behavioral markers of a developmental shift in the temporal dynamics of cognitive control. Proc Natl Acad Sci U S A 106:5529-5533.

Conway ARA, Kane MJ, Engle RW (2003) Working memory capacity and its relation to general intelligence. Trends Cogn Sci 7:547-552.

Cowan N (2005) Working memory capacity: essays in cognitive psychology. New York: Psychology Press.

Cowan N, Towse JN, Hamilton Z, Saults JS, Elliott EM, Lacey JF, Moreno MV, Hitch GJ (2003) Children's working-memory processes: a response-timing analysis. J Exp Psychol Gen 132:113-132.

Crone EA, Wendelken C, Donohue S, van Leijenhorst L, Bunge SA (2006) Neurocognitive development of the ability to manipulate information in working memory. Proc Natl Acad Sci U S A 103:9315-9320.

Curtis CE, D'Esposito M (2003) Persistent activity in the prefrontal cortex during working memory. Trends Cogn Sci 7:415-423.

Davidson MC, Amso D, Anderson LC, Diamond A (2006) Development of cognitive control and executive functions from 4 to 13 years: evidence from manipulations of memory, inhibition, and task switching. Neuropsychologia 44:2037-2078.
Eacott MJ, Gaffan D, Murray EA (1994) Preserved recognition memory for small sets, and impaired stimulus identification for large sets, following rhinal cortex ablations in monkeys. Eur J Neurosci 6:1466-1478.

Fair DA, Dosenbach NUF, Church JA, Cohen AL, Brahmbhatt S, Miezin FM, Barch DM, Raichle ME, Petersen SE, Schlaggar BL (2007) Development of distinct control networks through segregation and integration. Proc Natl Acad Sci U S A 104:13507-13512.

Fisher R (1921) On the "probable error"' of a coefficient of correlation deduced from a small sample. Metron 1:3-32.

Friston KJ, Frith CD, Liddle PF, Frackowiak RS (1991) Comparing functional (PET) images: the assessment of significant change. J Cereb Blood Flow Metab 11:690-699.

Friston KJ, Frith CD, Frackowiak RSJ, Turner R (1995a) Characterizing dynamic brain responses with fMRI: a multivariate approach. Neuroimage 2:166-172.

Friston KJ, Frith CD, Turner R, Frackowiak RSJ (1995b) Characterizing evoked hemodynamics with fMRI. Neuroimage 2:157-165.

Gathercole SE (1999) Cognitive approaches to the development of shortterm memory. Trends Cogn Sci 3:410-419.

Gogtay N, Nugent TF 3rd, Herman DH, Ordonez A, Greenstein D, Hayashi KM, Clasen L, Toga AW, Giedd JN, Rapoport JL, Thompson PM (2006) Dynamic mapping of normal human hippocampal development. Hippocampus 16:664-672.

Goldman-Rakic PS (1987) Circuitry of primate prefrontal cortex and regulation of behavior by representational memory. In: Handbook of physiology, the nervous system, higher functions of the brain (Plum F, ed), pp 373-417. Bethesda, MD: American Physiological Society.

Hannula DE, Tranel D, Cohen NJ (2006) The long and the short of it: relational memory impairments in amnesia, even at short lags. J Neurosci 26:8352-8359.

Klingberg T, Forssberg H, Westerberg H (2002) Increased brain activity in frontal and parietal cortex underlies the development of visuospatial working memory capacity during childhood. J Cogn Neurosci 14:1-10.

Lenroot RK, Giedd JN (2006) Brain development in children and adolescents: insights from anatomical magnetic resonance imaging. Neurosci Biobehav Rev 30:718-729.

Lenroot RK, Giedd JN (2010) Sex differences in the adolescent brain. Brain Cogn 72:46-55.

Luciana M, Nelson CA (1998) The functional emergence of prefrontallyguided working memory systems in four- to eight-year-old children. Neuropsychologia 36:273-293.

Luna B, Garver KE, Urban TA, Lazar NA, Sweeney JA (2004) Maturation of cognitive processes from late childhood to adulthood. Child Dev 75:1357-1372.

Mitchell KJ, Johnson MK, Raye CL, D’Esposito M (2000) fMRI evidence of age-related hippocampal dysfunction in feature binding in working memory. Brain Res Cogn Brain Res 10:197-206.

O'Hare ED, Lu LH, Houston SM, Bookheimer SY, Sowell ER (2008) Neurodevelopmental changes in verbal working memory load-dependency: an fMRI investigation. Neuroimage 42:1678-1685.

Olesen PJ, Macoveanu J, Tegnér J, Klingberg T (2007) Brain activity related to working memory and distraction in children and adults. Cereb Cortex 17:1047-1054.

Piekema C, Kessels RPC, Mars RB, Petersson KM, Fernández G (2006) The right hippocampus participates in short-term memory maintenance of object-location associations. Neuroimage 33:374-382.

Ramscar M, Gitcho N (2007) Developmental change and the nature of learning in childhood. Trends Cogn Sci 11:274-279.

Ranganath C, Blumenfeld RS (2005) Doubts about double dissociations between short- and long-term memory. Trends Cogn Sci 9:374-380.

Ranganath C, D'Esposito M (2001) Medial temporal lobe activity associated with active maintenance of novel information. Neuron 31:865-873.

Rissman J, Gazzaley A, D’Esposito M (2004) Measuring functional connectivity during distinct stages of a cognitive task. Neuroimage 23:752-763.

Rissman J, Gazzaley A, D’Esposito M (2008) Dynamic adjustments in prefrontal, hippocampal, and inferior temporal interactions with increasing visual working memory load. Cereb Cortex 18:1618-1629. 
Rypma B, D’Esposito M (2000) Isolating the neural mechanisms of agerelated changes in human working memory. Nat Neurosci 3:509-515.

Scherf KS, Sweeney JA, Luna B (2006) Brain basis of developmental change in visuospatial working memory. J Cogn Neurosci 18:1045-1058.

Sheppard LD, Vernon PA (2008) Intelligence and speed of informationprocessing: a review of 50 years of research. Pers Individ Dif 44:535551.

Sheridan MA, Hinshaw S, D'Esposito M (2007) Efficiency of the prefrontal cortex during working memory in attention-deficit/hyperactivity disorder. J Am Acad Child Adolesc Psychiatry 46:1357-1366.

Sowell ER, Thompson PM, Leonard CM, Welcome SE, Kan E, Toga AW (2004) Longitudinal mapping of cortical thickness and brain growth in normal children. J Neurosci 24:8223-8231.

Sternberg S (1969) Memory-scanning: mental processes revealed by reaction-time experiments. Am Scientist 57:421-457.

Thomas KM, King SW, Franzen PL, Welsh TF, Berkowitz AL, Noll DC,
Birmaher V, Casey BJ (1999) A developmental functional MRI study of spatial working memory. Neuroimage 10:327-338.

Thomason ME, Race E, Burrows B, Whitfield-Gabrieli S, Glover GH, Gabrieli JD (2009) Development of spatial and verbal working memory capacity in the human brain. J Cogn Neurosci 21:316-332.

Thompson-Schill SL, Ramscar M, Chrysikou EG (2009) Cognition without control: when a little frontal lobe goes a long way. Curr Dir Psychol Sci 18:259-263.

Tsujimoto S, Yamamoto T, Kawaguchi H, Koizumi H, Sawaguchi T (2004) Prefrontal cortical activation associated with working memory in adults and preschool children: an event-related optical topography study. Cereb Cortex 14:703-712.

Unsworth N, Redick TS, Heitz RP, Broadway JM, Engle RW (2009) Complex working memory span tasks and higher-order cognition: a latentvariable analysis of the relationship between processing and storage. Memory 17:635-654. 